\title{
Identification of Optimal Printing Conditions for Laser Printing of Alginate Tubular Constructs
}

\author{
Ruitong Xiong, Zhengyi Zhang, and Yong Huang* \\ Department of Mechanical and Aerospace Engineering, University of Florida, Gainesville, FL \\ 32611, USA \\ rtxiong@ufl.edu, zhengyz@ufl.edu,yongh@ufl.edu
}

\begin{abstract}
Three-dimensional (3D) laser-assisted orifice-free printing technique has emerged as a promising approach for the fabrication of tissue constructs. For the better adoption of this technique, this study has investigated the effects of operating conditions, including the laser fluence and receiving substrate velocity, on the printing quality in terms of whether printed features are well-defined or not. Four main morphologies of printed lines before gelation have been identified during line printing: isolated droplets, discontinuous segments, well-defined lines, and over-printed lines. The $2,125 \mathrm{~mJ} / \mathrm{cm}^{2}$ laser fluence and $100 \mathrm{~mm} / \mathrm{min}$ substrate velocity conditions, corresponding to a 0.42 overlap ratio, have been identified as a combination of optimal printing conditions by using an $8 \%$ alginate solution under a $10 \mathrm{~Hz}$ laser repetition rate. Alginate straight and bifurcated $Y$-shaped tubes have been successfully printed by applying the identified optimal printing conditions.
\end{abstract}

Keywords: Laser printing, optimal printing conditions, sodium alginate

\section{Introduction}

Tissue substitutes, including transplantable autograft and allograft tissues, have been widely utilized to recover or repair acute injury, chronic diseases, or congenital defects. However, such practices are usually limited by various hurdles such as pathogen transfer, immune rejection, high cost, and especially the donor shortage. Fortunately, organ printing, a layer-by-layer bioprinting technique, has emerged to tackle some of these hurdles by fabricating three-dimensional (3D) tissues/organs using patient's cells based on the computer-aided model of patient-specific organs (Ringeisen et al., 2013).

Comparing with scaffold-based tissue engineering approaches, 3D bioprinting such as jet-based laser printing (Riggs et al., 2011) and inkjet printing (Boland et al., 2007) offers a potentially scalable technology to fabricate heterogeneous structures from various cell types. Generally, bioprinting can be implemented in two ways: orifice-based and orifice-free. Nozzle-based bioprinting including inkjet

${ }^{*}$ Corresponding author

(C) 2015. This manuscript version is made available under the Elsevier user license http://www.elsevier.com/open-access/userlicense/1.0/ 
printing is a common orifice-based approach which has been successfully applied to print simple (Boland et al., 2006) (Nishiyama et al., 2009) as well as complex cellular tubes (Xu et al., 2012) (Christensen et al, 2015), and the droplet formation performance during the inkjet printing of cell-laden bioinks has been extensively investigated too (Xu et al., 2014). Unfortunately, orifice-based printing may experience a great difficulty in printing highly viscous biological materials which results in nozzle clogging during printing. For example, only sodium alginate with concentrations lower than $2 \%$ is recommended for inkjetting (Norman and Desai, 2006) (Herran and Huang, 2012a) (Herran et al., 2012b). As such, orifice-free techniques are expected for the printing of viscous biomaterials and biological materials or bioinks with a high cell density, which are common constituents of many biological constructs.

Of various orifice-free printing approaches, laser-assisted printing/direct writing, developed based on modified laser-induced forward transfer (LIFT), has been utilized to print and pattern different materials including biomaterials and biological materials (Barron et al., 2004) (Ringeisen et al., 2004) (Barron et al., 2005) (Lin et al., 2009a) (Lin et al., 2010) (Koch et al., 2010) (Schiele et al., 2010) (Lin and Huang, 2011) (Riggs et al., 2011) (Yan et al., 2013). During a typical modified LIFT-based laser direct-write process (Lin et al., 2009b), focused laser pulses are directed through a quartz ribbon which has a coating to be printed on the other side. These pulses are then absorbed by a sacrificial energy-absorbing layer or matrix of the coating, causing extremely localized heating and sublimation of a small portion of the coating. It leads to the formation of small vapor bubble(s), and the resulting bubble(s) further expand and may eject part of the coating material as a droplet for deposition or patterning. There are four main sequential steps during a typical laser printing process, namely, laser-matter interaction, bubble formation and expansion, jet/droplet formation, and jet/droplet landing.

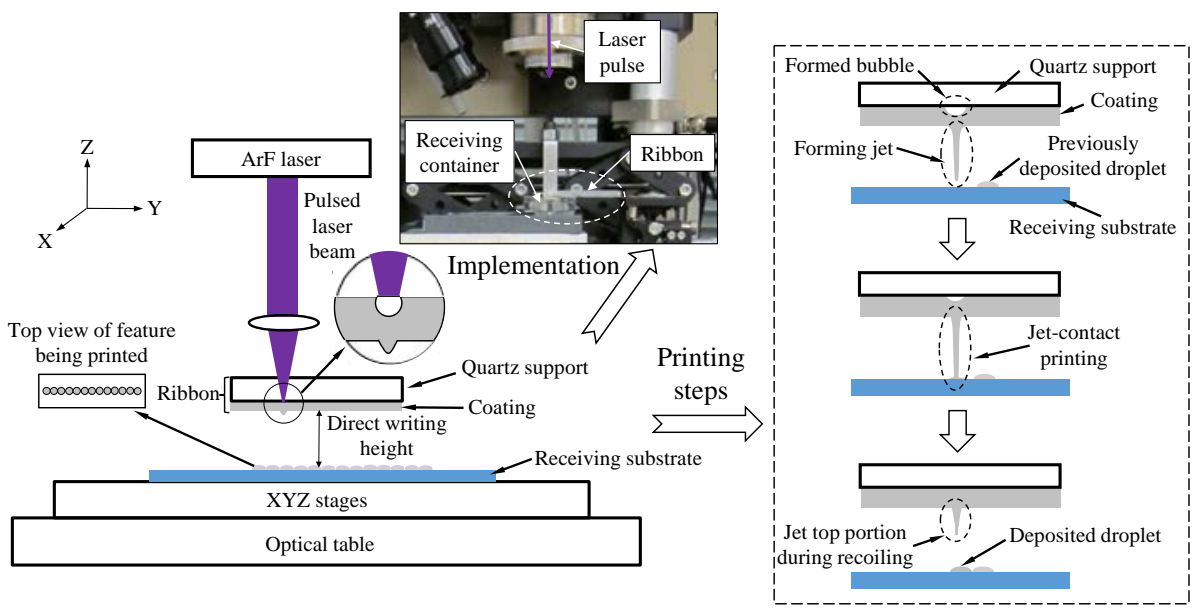

Figure 1: Laser printing schematic and jet-contact printing steps

Laser printing is usually implemented as matrix-assisted pulsed-laser evaporation direct-write (MAPLE DW) (Lin et al., 2009b) as shown in Figure 1. Once a laser pulse-induced bubble (Xiong et al., 2015) is formed during laser printing, the bubble may lead to a forming jet. As the jet further develops, it may contact the receiving substrate, which can be a previously printed layer, before breaking up into droplet(s) depending on the direct writing height, and the top portion of the jet may recoil back to the coating of the ribbon. Such a jet-contact printing scenario usually leads to a good feature resolution. Alternatively, the jet may break up into droplet(s) first and then land onto the substrate. Once deposited, a droplet with a landing velocity may impact (Wang et al., 2008) and further spread over the substrate. Ideally, successively printed droplets should gel/solidify and coalesce into an integral, continuous feature. Such a feature is continuously deposited at the same elevation with the help of support material as needed, 
and once a layer is formed, the receiving substrate moves downwards by a distance of layer thickness inside a container until a construct is printed as described by Yan et al. (Yan et al., 2013).

In addition to being utilized to print various cells (Barron et al., 2004) (Ringeisen et al., 2004) (Lin et al., 2009a) (Lin et al., 2010) (Koch et al., 2010), laser printing has been pioneered to make twodimensional (2D) and 3D constructs (Koch et al., 2009) (Gruene et al., 2011) (Yan et al., 2013). Of various laser printed 3D printed constructs, their surface finish and dimensional accuracy are still to be further improved by optimizing laser printing conditions for any given material being printed. As a logical step to solve this printing quality challenge, laser line printing should be first carefully investigated since printed lines are basic building blocks to form each layer, eventually leading to a layer-by-layer fabricated construct.

Line quality during laser printing has been a research subject of numerous studies. For example, the printing of conductive nanoparticles has been investigated by varying the laser pulse energy and donorreceiver substrate separation to analyze their effects on transferred droplets (Rapp et al., 2011); the influence of droplet spacing on the morphology of printed lines has been researched during the laser printing of glycerol solution (Palla-Papavlu et al., 2013). Unfortunately, there is still no work to study the laser line printing of viscoelastic solutions/suspensions, which represent most common bioinks. The fluid viscoelasticity usually significantly influences the jet/droplet formation process as well as the droplet spreading process after landing. Furthermore, further research on applying optimized printing conditions to laser print 3D constructs is still missing.

The objective of this study is to experimentally investigate operating conditions on the line printing quality in terms of whether printed features are well-defined or not during the laser printing of viscoelastic alginate solution and further apply identified optimal conditions for the fabrication of 3D alginate tubular constructs. In particular, laser fluence and substrate velocity have been selected as two operating conditions of interest herein. Alginate, especially sodium alginate, has been widely used as a constituent of bioinks in bioprinting, and it can be chemically modified for better cell adhesion capacity and biodegradability. As such, sodium alginate has been chosen to prepare the viscoelastic bioink to be printed in this study. While sodium alginate solution has been printed herein, the resulting knowledge may be applicable to the printing of other bioinks.

\section{Experiment Setup and Design}

\subsection{Experiment Setup}

Laser printing was implemented as MAPLE DW to investigate the effects of laser fluence and substrate velocity on the printing quality and further fabricate 3D alginate tubular constructs. The MAPLE DW apparatus consisted of an ExciStar argon fluoride (ArF) 193 nm, 12 ns full-width half-maximum eximer laser (Coherent, Santa Clara, CA), a laser beam deliver system, an alginate coated ribbon, and a moving substrate controlled by XYZ stages (Aerotech, Pittsburg, PA). In particular, the printing setup is illustrated in Figure 1.

In this study the laser spot size was controlled as $150 \mu \mathrm{m}$ in diameter, the laser repetition rate was 10 $\mathrm{Hz}$, and ultraviolet (UV) fused silica quartz (Edmund Optics, Barrington, NJ) with 85\% transmittance for $193 \mathrm{~nm}$ laser was used as the quartz support for the ribbon. The coating was prepared using a film applicator (MTI, Richmond, CA), resulting in a ribbon coating with a thickness of approximately $50 \mu \mathrm{m}$. The laser fluence was measured using a FieldMax laser power/energy meter (Coherent, Santa Clara, CA), the ribbon motion was controlled by computer-controlled motion stages (Thorlabs, Newton, NJ) to continuously expose new coating materials for laser printing, and printed features were examined using an optical microscope and analyzed using ImageJ (National Institute of Health, Bethesda, Maryland). Herein 
the direct writing height, defined as the distance between the ribbon and the receiving substrate, was set at $2 \mathrm{~mm}$ based on the preliminary testing to achieve a jet-contact printing condition, which usually leads to a good printing quality while avoiding an undesirable contact between the coating material and the crosslinking solution. Such a $2.0 \mathrm{~mm}$ direct writing height was also adopted in a previous laser printing study (Yan et al., 2013).

\subsection{Materials and Design of Experiments}

Sodium alginate (NaAlg) (Sigma-Aldrich, St. Louis, MO), which has a molecular weight range of 20$40 \mathrm{kDa}$, was mixed with deionized water to prepare the sodium alginate solution with a concentration of $8 \%(\mathrm{w} / \mathrm{v})$. The $8 \%(\mathrm{w} / \mathrm{v})$ sodium alginate solution was chosen for its overall stable jet formation performance, so only the laser fluence and substrate velocity were chosen as control variables in this study. It should be pointed out that both the sodium alginate concentration and the direct writing height are also the important material property and printing condition, respectively, which should be further investigated in a systematic way for robust laser printing. In addition, calcium chloride dehydrate (Sigma-Aldrich, St. Louis, MO), a cross-linking agent for sodium alginate, was used to prepare the cross-linking solution as a source of calcium cations to initiate gelation once in contact with alginate radicals. During structural printing such as the printing of $Y$-shaped constructs, the calcium chloride solution also behaved as the support liquid for the printing of overhanging and spanning features (Xu et al., 2013) (Christensen et al., 2015).

\begin{tabular}{crr}
\hline $\begin{array}{l}\text { Actual average } \\
\text { laser fluence } \\
\left(\mathrm{mJ} / \mathrm{cm}^{2}\right)\end{array}$ & Substrate velocity (mm/min) & Droplet spacing (mm) \\
\hline 1,445 & $40,60,80,100,120$ & $0.07,0.10,0.13,0.17,0.20$ \\
1,615 & $40,60,80,100,120$ & $0.07,0.10,0.13,0.17,0.20$ \\
1,785 & $60,80,100,120,140$ & $0.10,0.13,0.17,0.20,0.23$ \\
1,955 & $60,80,100,120,140$ & $0.10,0.13,0.17,0.20,0.23$ \\
2,125 & $60,80,100,120,140,160,180$ & $0.10,0.13,0.17,0.20,0.23,0.27,0.30$ \\
\hline
\end{tabular}

Table 1: Laser fluence and substrate velocity combinations tested and resulting droplet spacing

Table 1 shows the laser fluence and substrate velocity combinations adopted in this laser printing study. While the laser fluence directly influences the diameter of forming droplets, the center-to-center droplet spacing of two adjacent droplets is determined by the substrate velocity and laser repetition rate. The laser fluence range shown in Table 1 was selected based on a preliminary time-resolved study to investigate the jet and droplet formation performance to have well-defined jets during the laser printing of viscoelastic alginate solutions without the presence of substrate. For a given laser fluence, the permissible substrate velocity for quality printing should be selected to guarantee a connected line feature.

In order to quantitatively analyze the difference between the morphologies of printed lines, an overlap ratio $\lambda$ is used to quantify the overlap between two adjacent droplets:

$$
\lambda=1-\frac{L}{D_{d}}
$$


where $L$ is the center-to-center droplet spacing, and $D_{d}$ is the deposited droplet diameter. $L$ can be calculated based on the substrate velocity $V_{s}$ and the laser repetition rate $f$ as $\frac{v_{s}}{f}$, and the resulting $L$ in this study are shown in Table 1 . For a complete overlap, when droplet centers coincide, $\lambda=1$, while $\lambda<0$ for there is no overlap.

\section{Experiment Results and Discussion}

\subsection{Morphologies of Printed Lines}

During a typical laser printing process, four main morphologies of printed lines before gelation have been identified depending on printing conditions: (a) isolated droplets, (b) discontinuous segments, (c) well-defined lines, and (d) over-printed lines as shown in Figure 2, which can be classified into four regimes, respectively. For the first regime (Figure 2(a)), droplets are deposited as isolated features without connecting to each other to form a line, which is mainly due to their small droplet size and/or high substrate velocity. When the droplet size increases and/or the substrate velocity decreases, deposited droplets start connecting to each other, appearing as some discontinuous line segments as the second regime (Figure 2(b)). When the droplet size further increases and/or the substrate velocity further decreases, a well-defined, continuous line is formed as shown in Figure 2(c) (third regime), and such as a line has a relatively uniform line width and its edge is smooth. This regime is expected for construct printing since its printing quality facilitates the fabrication of 3D constructs. As such, the associated laser fluence and substrate velocity ranges must be identified first as optimal printing conditions for laser printing. However, if the droplet size further increases or the substrate velocity further decreases, printed lines are still continuous, but some portions are over-printed and swollen (Fourth regime, Figure 2(d)). The resulting line edge is jagged, which is not ideal for construct fabrication.

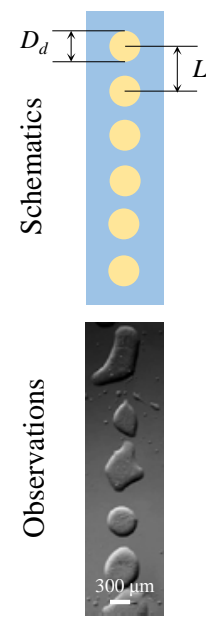

(a)

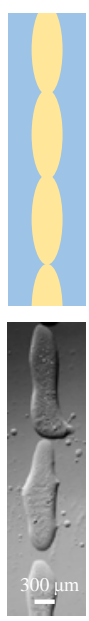

(b)

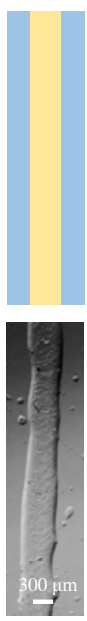

(c)

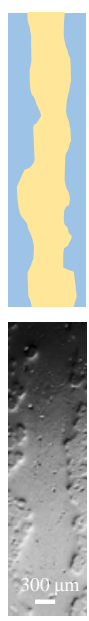

(d)

Figure 2: Four morphologies of printed lines: (a) isolated droplets, (b) discontinuous segments, (c) welldefined lines, and (d) over-printed lines 


\subsection{Determination of Optimal Printing Conditions}

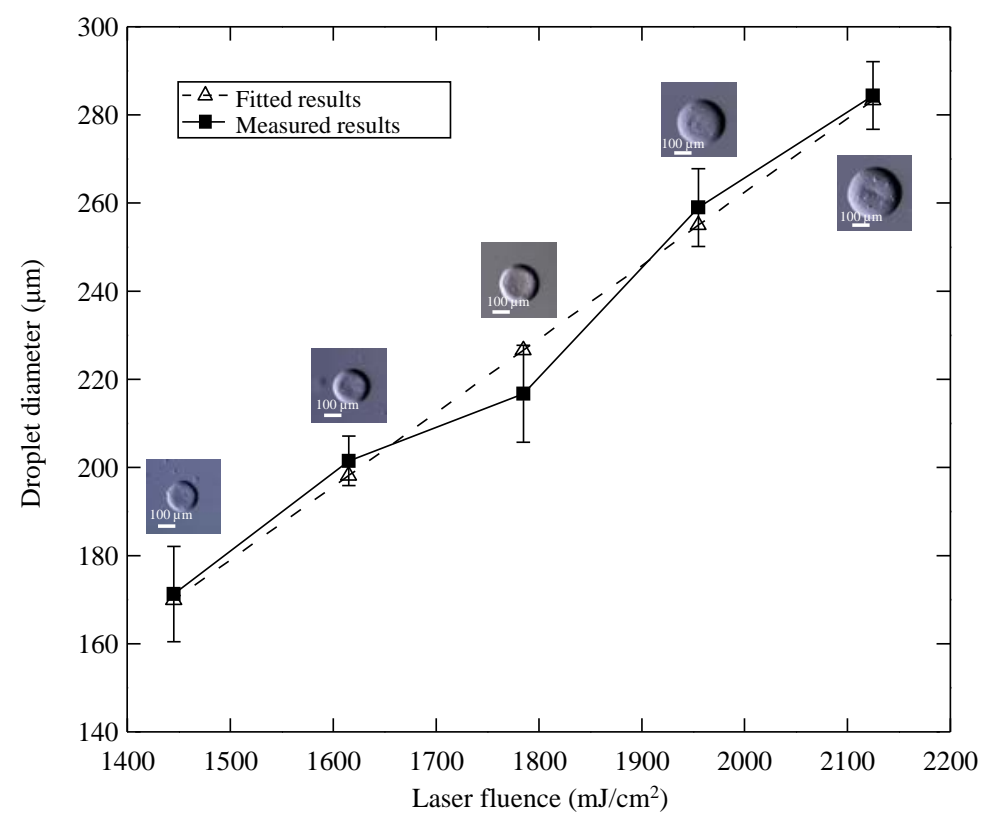

Figure 3: Droplet diameter as a function of laser fluence when printing an 8\% NaAlg solution (Error bar represents \pm 1 standard deviations)

As observed, the droplet diameter increases as the laser fluence increases based on the average of five measurements of individual droplets. It should be noted that the droplet diameter was measured after deposition and this gave enough time for droplets to spread, which usually finishes on the order of $100 \mu \mathrm{s}$ (Duocastella et al., 2010). As shown in Figure 3, their relationship can be reasonably approximated as a linear fitting as follows:

$$
D_{d}=0.17 \phi-71.30
$$

where $\phi$ is the applied laser fluence.

Then Equation (1) can be rewritten in terms of $V_{S}, f(10 \mathrm{~Hz}$ in this study) and $\phi$ as follows:

$$
\lambda=1-\frac{v_{s} / f}{0.17 \phi-71.30}=1-\frac{v_{s}}{1.7 \phi-713.0}
$$

where the overlap ratio increases with the laser fluence and decreases with the substrate velocity.

Figure 4 shows the effect of the laser fluence on the morphology of printed lines under a fixed substrate velocity (100 mm/min). For laser fluences from $1,445 \mathrm{~mJ} / \mathrm{cm}^{2}$ to $1,785 \mathrm{~mJ} / \mathrm{cm}^{2}$, the printed lines can be classified as discontinuous segments; for laser fluences from $1,785 \mathrm{~mJ} / \mathrm{cm}^{2}$ to $2,125 \mathrm{~mJ} / \mathrm{cm}^{2}$, where the overlap ratio is 0.36 and 0.42 for those two laser fluences, the printed lines are well-defined lines. For laser fluences smaller than $1,445 \mathrm{~mJ} / \mathrm{cm}^{2}$, there is no material transferred while for laser fluences larger than $2,125 \mathrm{~mJ} / \mathrm{cm}^{2}$, no good jets can be formed according to a preliminary time-resolved study. 


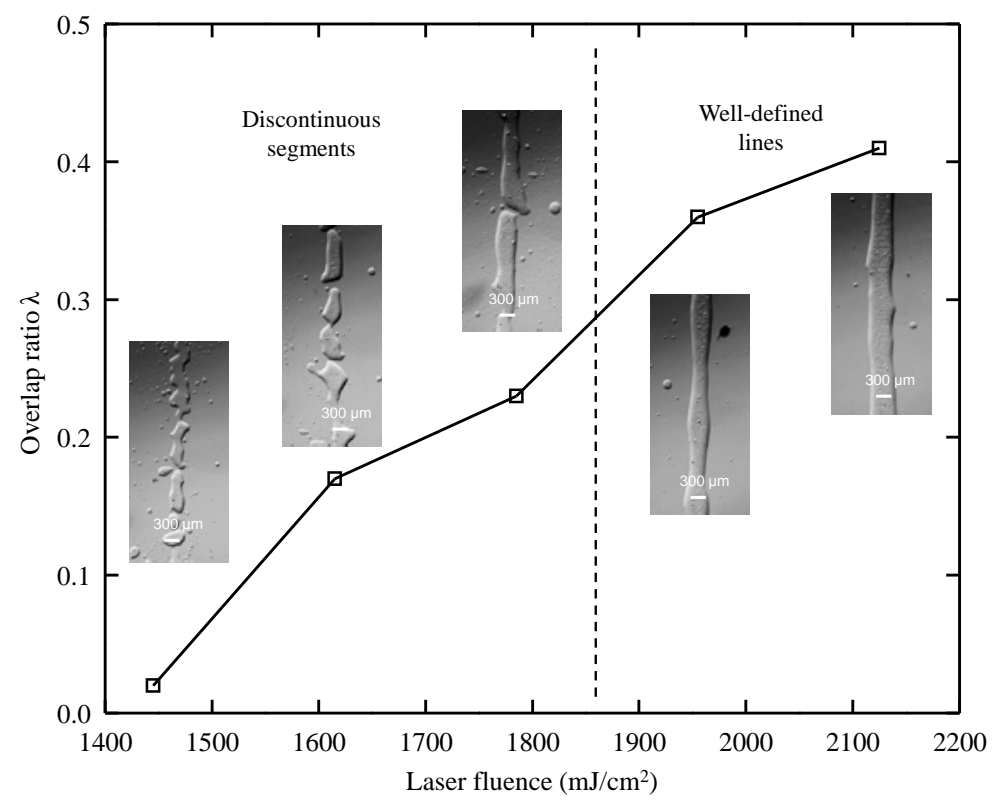

Figure 4: Line morphology as a function of laser fluence (substrate velocity $=100 \mathrm{~mm} / \mathrm{min}$ )

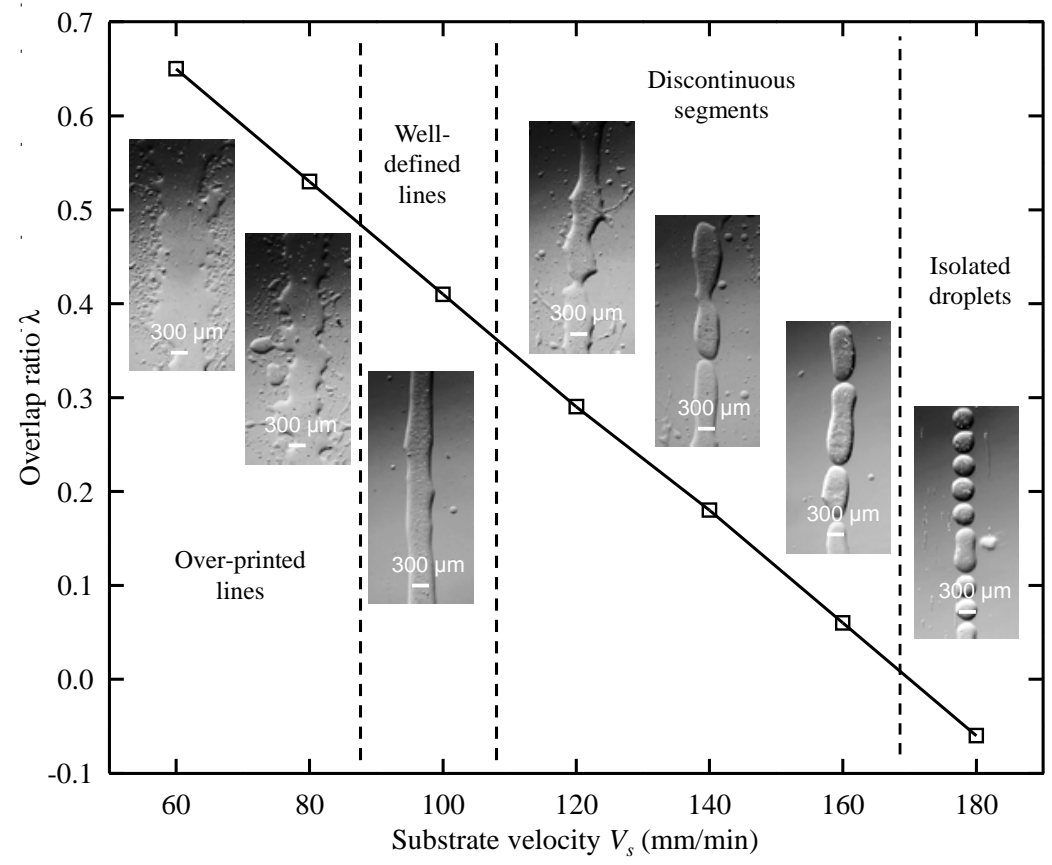

Figure 5: Line morphology as a function of substrate velocity (laser fluence $=2,125 \mathrm{~mJ} / \mathrm{cm}^{2}$ )

The effect of the substrate velocity on the morphology of printed lines can be examined under a fixed laser fluence of $2,125 \mathrm{~mJ} / \mathrm{cm}^{2}$ as shown in Figure 5 . Under the given laser fluence, the size of deposited 
droplets is constant. It is found that the morphology of printed lines changes from over-printed lines to well-defined lines to discontinuous segments to isolated droplets as the substrate velocity increases. At the substrate velocity of $100 \mathrm{~mm} / \mathrm{min}$, well-defined lines can be printed, corresponding to an overlap ratio of 0.42 .

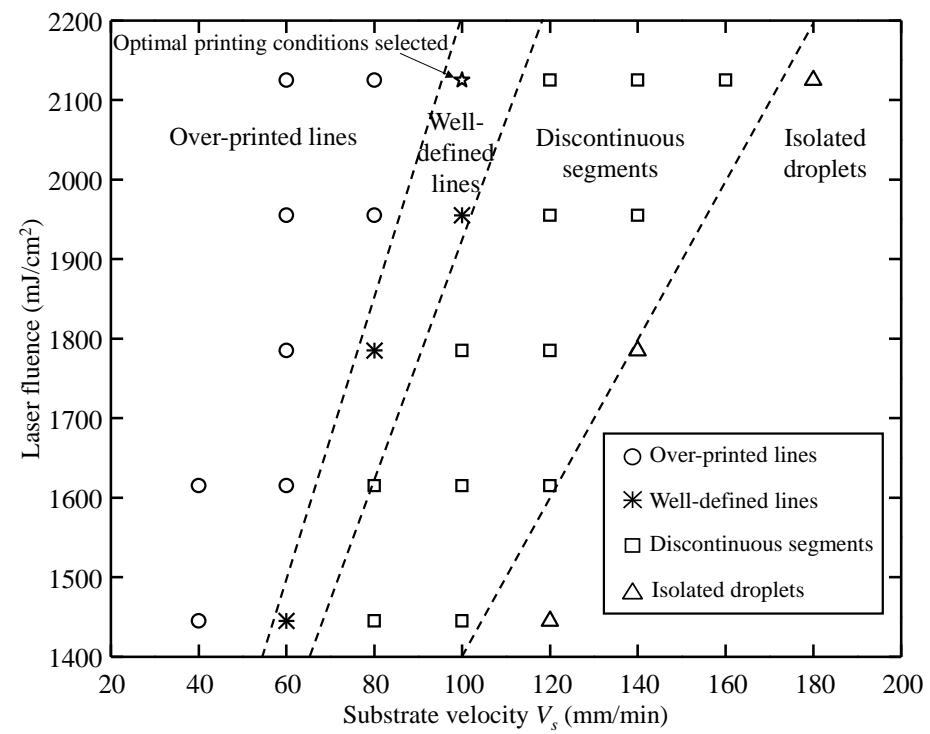

Figure 6: Line morphology as a function of substrate velocity and laser fluence

As seen from Figures 4 and 5, there are different regimes determining the morphology of printed lines under a given combination of laser fluence and substrate velocity, which is shown in Figure 6. The dash lines, corresponding to an overlap ratio, respectively (0.00, 0.35 , and 0.45 herein), are drawn based on Equation (3) and delineate the different printing regimes from each other. To achieve a better printing quality, the combination of laser fluence and substrate velocity can be selected by using Figure 6 . Based on the experimental results herein, the $2,125 \mathrm{~mJ} / \mathrm{cm}^{2}$ laser fluence and $100 \mathrm{~mm} / \mathrm{min}$ substrate velocity conditions, corresponding to a 0.42 overlap ratio, may result in a good line printing quality and are identified as a combination of optimal printing conditions. This set of the optimal printing conditions is also depicted using a star in Figure 6. It should be pointed out that the laser fluence should be first selected to guarantee the formation of well-defined jets (Yan et al., 2012).

When comparing with those printed from glycerol solution (Palla-Papavlu et al., 2013), which is a typical viscous Newtonian fluid, the well-defined lines of viscoelastic sodium alginate solution herein are not as perfectly uniform in width. This is attributed to the development of elastic stress inside viscoelastic droplets as they begin to coalesce and retract (Zhou et al., 2010). For viscous fluids, the factor which may hinder the surface tension-driven coalescence is the viscous force only (Ristenpart et al., 2006). However, for viscoelastic fluids, in addition to the viscous force, the elastic force developed may also hamper the coalescent and retraction process of landed droplets which makes it harder to form smooth edges on the printed lines. As such, well-defined lines printed with viscoelastic sodium alginate solutions may appear less uniform. 


\section{Printing of Alginate Tubular Constructs}

The identified optimal printing conditions $\left(2,125 \mathrm{~mJ} / \mathrm{cm}^{2}\right.$ laser fluence and $100 \mathrm{~mm} / \mathrm{min}$ substrate velocity) have been further applied to print alginate tubular constructs by following the procedure as outlined by Yan et al. (Yan et al., 2013). It is assumed that the gelation doesn't significantly change the printing quality, which was confirmed in this study too.

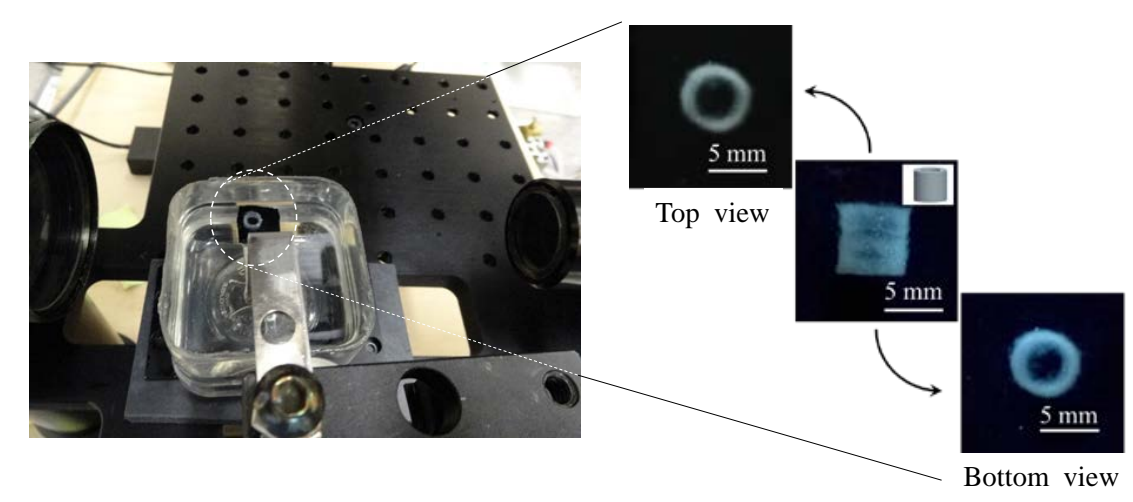

Figure 7: Alginate straight tube printed using optimal printing conditions

To mimic typical vascular constructs in organ printing, both straight and $Y$-shaped tubes were printed using a circular path for each layer. Figure 7 shows a representative alginate straight tube with a diameter of $5.0 \mathrm{~mm}$, a height of $5.0 \mathrm{~mm}$, which achieves a much better printing quality than those reported by Yan et al. (Yan et al., 2013). In particular, the surface finish of the printed tube herein is smoother, the tube ends are well defined, and the wall thickness is more uniform, demonstrating the effectiveness of optimal printing conditions during laser printing.

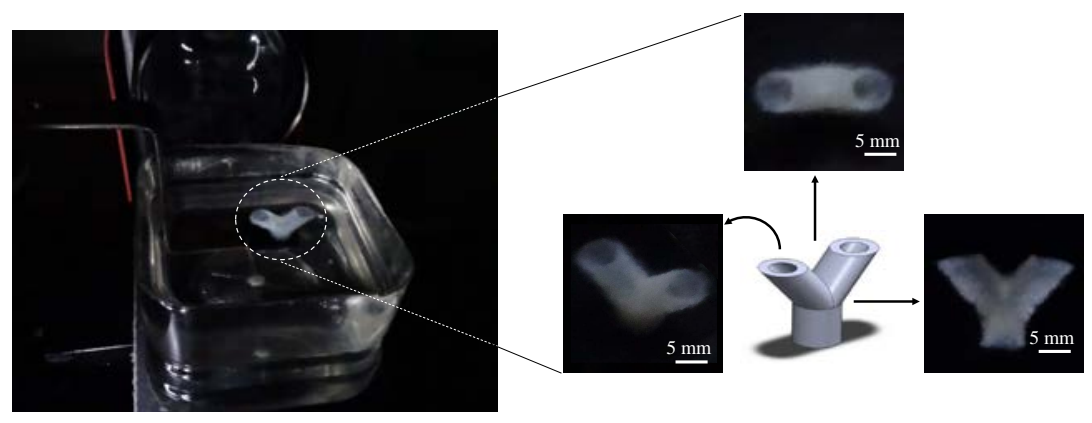

Figure 8: Alginate $Y$-shaped tube printed using optimal printing conditions

Figure 8 illustrates a representative alginate $Y$-shaped tube with a diameter of $5.0 \mathrm{~mm}$, a straight tube height of $4.0 \mathrm{~mm}$, an overhanging tube height of $5.5 \mathrm{~mm}$, and an overhanging angle of 45 degrees. This is the first reported 3D complex structure fabricated using laser printing. As aforementioned, the calcium chloride solution also behaved as the support liquid by providing a buoyant force for the printing of overhanging and spanning features. During the printing of the vertical bifurcation, a straight tube was first printed vertically, and it was gradually split into two inclined tubes layer-by-layer via a junction. This bifurcation fabrication is permissible by printing layers slightly overhanging from previous layers and eventually spanning the gap across the lumen of the straight tube by utilizing the buoyancy provided by 
the calcium chloride solution. While the tube printing quality can be further improved, both the constructs demonstrate a well-defined shape, illustrating promising applications of laser printing under optimal operating conditions.

Under the printing conditions for well-defined lines $(2,125 \mathrm{~mJ} / \mathrm{cm} 2$ laser fluence and $100 \mathrm{~mm} / \mathrm{min}$ substrate velocity) for construct printing, the measured line width and thickness before gelation are 458.8 $\pm 16.9 \mu \mathrm{m}$ and $36.6 \pm 4.6 \mu \mathrm{m}$, respectively based on the average of five measurements. The construct wall thickness are approximately $1,200.0 \mu \mathrm{m}$ for the straight tube and $1,500.0 \mu \mathrm{m}$ for the Y-shaped tube (measured based the bottom straight portion), respectively, and the layer thicknesses is $30.0 \mu \mathrm{m}$. While they are directly correlated, the construct wall width and layer thickness are not the exactly same as those of well-defined lines before gelation as observed (Section 3) due to the swelling effect of the alginate gelation process as well as the possible elastic deformation of lateral spreading and vertical compression of gelled layers. In addition, the expected deposition location of each droplet may be affected by the substrate movement-induced construct shaking, resulting in a thicker construct wall. As such, the step size of the downward movement of the receiving substrate was carefully controlled at $30.0 \mu \mathrm{m}$ to match the layer thickness of printed constructs. Since the wall thickness of the printed tubular constructs herein is larger than the width of the 2D lines before gelation. For the fabrication of any designed structure, the swelling effect should be especially considered to guarantee that the final inner diameter meets physiological and anatomical requirements.

\section{Conclusions and Future Work}

Laser printing has been first investigated during line printing to identify optimal printing conditions. Four types of printed lines have been identified depending on printing conditions: isolated droplets, discontinuous segments, well-defined lines, and over-printed lines. The $2,125 \mathrm{~mJ} / \mathrm{cm}^{2}$ laser fluence and $100 \mathrm{~mm} / \mathrm{min}$ substrate velocity conditions, corresponding to a 0.42 overlap ratio, have been identified as a combination of optimal printing conditions by using an $8 \%$ alginate solution under a $10 \mathrm{~Hz}$ laser repetition rate. The identified printing conditions have been implemented to successfully print alginate straight tube and $Y$-shaped constructs. It is noted that $8 \%$ alginate is way too high for cell printing. As reported (Gudapati et al., 2014), the post-printing sodium alginate concentration is significantly influenced by the sodium alginate concentration, and even when using a 3\% sodium alginate solution, the resulting cell viability is barely above $50 \%$. It is expected that the methodology developed herein applies to the process optimization of the printing of lowly concentrated alginate-cell suspensions under a sacrificial energy absorbing layer such as gelatin as well as other soft non-cellular structures.

For better applications of laser bioprinting, future work may focus on: (1) the identification of permissible range of overlap ratio for good printing quality for a given material, (2) the printing of complex cellular constructs and the investigation of the printing process-induced injury and influence, (3) the evaluation of mechanical integrity such as the interfacial bonding strength of printed constructs using tensile and compression testing, (4) the automation of the laser printing system for scale-up fabrication, (5) the application of energy absorption layer on the ribbon to further improve the printing quality, and (6) detailed analysis and modeling of the failure mechanism during the printing of overhang and spanning branches for better structural printing.

\section{Acknowledgements}

The study was partially supported by the National Science Foundation (CMMI-1314830 and CAREER 1321271). The experimental assistance from Brian Davis is also highly appreciated. 


\section{References}

Barron JA, Ringeisen BR, Kim H, Spargo BJ and Chrisey DB. Application of laser printing to mammalian cells. Thin Solid Films 2004; 453: 383-387.

Barron JA, Young HD, Dlott DD, Darfler MM, Krizman DB and Ringeisen, BR. Printing of protein microarrays via a capillary - free fluid jetting mechanism. Proteomics 2005; 5(16): 4138-4144.

Brown MS, Brasz CF, Ventikos Y and Arnold CB. Impulsively actuated jets from thin liquid films for high-resolution printing applications. Journal of Fluid Mechanics 2012; 709: 341-370.

Christensen K, Xu C, Chai W, Zhang Z, Fu J and Huang Y. Freeform inkjet printing of cellular structures with bifurcations. Biotechnology and Bioengineering 2015; In press. DOI: 10.1002/bit.25501.

Duocastella M, Fernández-Pradas JM, Morenza JL and Serra P. Time-resolved imaging of the laser forward transfer of liquids. Journal of Applied Physics 2009; 106(8): 084907.

Duocastella M, Fernández-Pradas JM, Morenza, JL and Serra P, Sessile droplet formation in the laserinduced forward transfer of liquids: a time-resolved imaging study. Thin Solid Films 2010; 518(18): 5321-5325.

Gruene M, Pflaum M, Hess C, Diamantouros S, Schlie S, Deiwick A and Chichkov B. Laser printing of three-dimensional multicellular arrays for studies of cell-cell and cell-environment interactions. Tissue Engineering Part C: Methods 2011; 17(10): 973-982.

Gudapati H, Yan J, Huang Y and Chrisey DB. Alginate gelation-induced cell death during laser-assisted cell printing. Biofabrication 2014; 6(3): 035022.

Herran CL and Huang Y. Alginate microsphere fabrication using bipolar wave-based drop-on-demand jetting. Journal of manufacturing processes 2012a; 14(2): 98-106.

Herran, CL, Huang Y and Chai W. Performance evaluation of bipolar and tripolar excitations during nozzle-jetting-based alginate microsphere fabrication. Journal of Micromechanics and Microengineering 2012b; 22(8): 085025.

Koch L, Kuhn S, Sorg H, Gruene M, Schlie S, Gaebel R and Chichkov. Laser printing of skin cells and human stem cells. Tissue Engineering Part C: Methods 2009; 16(5): 847-854.

Li R, Ashgriz N, Chandra S, Andrews JR and Williams J. Drawback during deposition of overlapping molten wax droplets. Journal of Manufacturing Science and Engineering 2008; 130(4): 041011.

Lin Y, Huang Y, Wang G, Tzeng TJ and Chrisey DB. Effect of laser fluence on yeast cell viability in laserassisted cell transfer. Journal of Applied Physics 2009a; 106(4): 043106.

Lin Y, Huang Y and Chrisey DB. Droplet formation in matrix-assisted pulsed-laser evaporation direct writing of glycerol-water solution. Journal of Applied Physics 2009b; 105(9): 093111.

Lin Y, Huang G, Huang Y, Tzeng TJ and Chrisey DB. Effect of laser fluence in laser-assisted direct writing of human colon cancer cell. Rapid Prototyping Journal 2010; 16(3): 202-208.

Lin Y and Huang Y. Laser-assisted fabrication of highly viscous alginate microsphere. Journal of Applied Physics 2011; 109(8): 083107.

Norman JJ and Desai TA. Methods for fabrication of nanoscale topography for tissue engineering scaffolds. Annals of biomedical engineering 2006; 34(1): 89-101.

Palla-Papavlu A, Córdoba C, Patrascioiu A, Fernández-Pradas JM, Morenza JL and Serra P. Deposition and characterization of lines printed through laser-induced forward transfer. Applied Physics A 2013; 110(4): 751-755.

Rapp L, Ailuno J, Alloncle AP and Delaporte P. Pulsed-laser printing of silver nanoparticles ink: control of morphological properties. Optics express 2011; 19(22): 21563-21574.

Riggs BC, Dias AD, Schiele NR, Cristescu R, Huang Y, Corr DT and Chrisey DB. Matrix-assisted pulsed laser methods for biofabrication. MRS Bulletin 2011; 36(12): 1043-1050.

Ringeisen BR, Kim H, Barron JA, Krizman DB, Chrisey DB, Jackman S and Spargo BJ. Laser printing of pluripotent embryonal carcinoma cells. Tissue Engineering 2004; 10(3-4): 483-491. 
Ringeisen BR, Pirlo RK, Wu PK, Boland T, Huang Y, Sun W and Chrisey DB. Cell and organ printing turns 15: diverse research to commercial transitions. MRS Bulletin 2013; 38(10): 834-843.

Ristenpart WD, McCalla PM, Roy RV and Stone HA. Coalescence of spreading droplets on a wettable substrate. Physical Review Letters 2006; 97(6): 064501.

Schiele NR, Corr DT, Huang Y, Raof NA, Xie Y and Chrisey DB. Laser-based direct-write techniques for cell printing. Biofabrication 2010; 2(3): 032001.

Wang W, Huang Y, Grujicic M and Chrisey DB. Study of impact-induced mechanical effects in cell direct writing using smooth particle hydrodynamic method. Journal of Manufacturing Science and Engineering 2008; 130(2): 021012.

Xiong R, Zhang Z, Shen J, Lin Y, Huang Y and Chrisey DB. Bubble formation modeling during laser direct writing of glycerol solutions. Journal of Micro and Nano-Manufacturing 2015; 3(1): 011004.

Xu C, Chai W, Huang Y and Markwald RR. Scaffold - free inkjet printing of three - dimensional zigzag cellular tubes. Biotechnology and Bioengineering 2012; 109(12): 3152-3160.

Xu C, Christensen K, Zhang Z, Huang Y, Fu J and Markwald RR. Predictive compensation-enabled horizontal inkjet printing of alginate tubular constructs. Manufacturing Letters 2013; 1(1): 28-32.

Xu C, Zhang M, Huang Y, Ogale A, Fu J and Markwald RR. Study of droplet formation process during drop-on-demand inkjetting of living cell-laden bioink. Langmuir 2014; 30(30): 9130-9138.

Yan J, Huang Y, Xu C and Chrisey DB. Effects of fluid properties and laser fluence on jet formation during laser direct writing of glycerol solution. Journal of Applied Physics 2012; 112(8): 083105.

Yan J, Huang Y and Chrisey DB. Laser-assisted printing of alginate long tubes and annular constructs. Biofabrication 2013; 5(1): 015002.

Zhou C, Yue P, Feng JJ, Ollivier-Gooch CF and Hu HH. 3D phase-field simulations of interfacial dynamics in Newtonian and viscoelastic fluids. Journal of Computational Physics 2010; 229(2): 498511. 